(Aus dem physiologischen Institut der Universität Königsberg i. Pr.)

\title{
Über indirekte Muskelreizung durch Stromstösse und Strompausen.
}

Von

M. Gildemeister (Strassburg i. E.) und O. Weiss (Königsberg i. Pr.).

(Mit 3 Textfiguren.)

Die Versuche, über die wir hier berichten, haben wir schon vor längerer Zeit im Königsberger physiologischen Institut angestellt. Sie wurden im Oktober 1902 von uns begonnen; der eine von uns konnte über einige Resultate derselben schon in der Sitzung der PhysikalischÖkonomischen Gesellschaft zu Köniqsberg vom 27. November 1902 berichten. Erst als wir unsere Arbeit im wesentlichen abgeschlossen batten, erhielten wir davon Kenntnis, dass G. Weiss ${ }^{1}$ ) sich kurz vorher mit demselben Problem beschäftigt hătte, und dass sich seine Ergebnisse mit demjenigen Teil unserer Arbeit deckten, der sich auf Stromstösse bezog.

Wir unterliessen darauf die ausführliche Veröffentlichung unserer Untersuchungen, in der Absicht, sie später im Verein mit verwandten Aufgaben wieder aufzunehmen. Der eine von uns bat in einer bald darauf erschienenen Arbeit ${ }^{2}$ ) auf die Resultate nur kurz hingewiesen. Diese kurze Bemerkung ist wohl, wie es auch nicht anders zu erwarten war, der Aufmerksamkeit von Keith Lukas entgangen; wenigstens erwähnt dieser Autor in seiner Arbeit über Strompausen ${ }^{g}$ ) nichts davon.

In neuester Zeit hat sich das Interesse der Physiologen, angeregt durch den Streit der Erregungstheorien, wieder der Reizung durch sehr flüchtige elektrische Vorgänge zugewandt. Da unsere Versuche mit sehr präzisen Hilfsmitteln angestellt sind und sich auf besonders kleine Zeiten erstrecken, so haben sie zweifellos einigen Wert als Material zur Prüfung dieser Theorien. Überdies ist unsere Absicht, verwandte Probleme gemeinsam zu verfolgen, durch äussere Üm-

1) G. Weiss, Arch. ital. de biol. t. 35 p. 413.

2) M. Gildemeister, Über ein mechanisches Modell eines Nervmuskelpräparates. Pflüger's Arch. Bd. 101 S. 57 unten und S. 60 oben.'

3) Keith Lukas, Journ. of physiol. vol. 35 p. 310. 1907.

E. Pflüger, Arehiv für Physiologie. Bd. 130. 
stände vorläufig vereitelt. Deshalb halten wir diese nachträgliche Veröffentlichung nicht für überflüssig. Entsprechend unserer Absicht, nur unser Material für andere zugänglich zu machen, werden wir uns auf kurze Mitteilung der Tatsachen beschränken, ohne uns an dieser Stelle auf theoretische Erörterungen einzulassen. Auch werden wir hier nicht zu den unterdessen erschienenen Arbeiten auf diesem Gebiete Stellung nebmen.

\section{Methodisches.}

Als Untersuchungsobjekt diente in allen Fällen das übliche Nervmuskelpräparat von Rana esculenta, das unter den bekannten Vorsichtsmaassregeln (unpolarisierbare Elektroden, Schutz gegen Austrocknung) gereizt wurde. Die Zuckungen des Muskels wurden teils durch Beobachtung, teils graphisch festgestellt.

Als Apparat zum Öffnen und Schliessen des elektrischen Stromes diente das Helmholtz'sche Pendel in der Edelmann'schen Ausführung, das wir schon bei Gelegenheit früherer Untersuchungen beschrieben haben ${ }^{1}$ ). Der von uns modifizierte Schliesskontakt lieferte ganz reine Schliessungen ohne jedes Klirren, wovon wir uns oft überzeugten. Die Momente der Stromöffnung und des Stromschlusses wurden durch Drehung einer feinen Schraube gegeneinander verschoben.

Die Präzision dieses Apparates kann auf einer sehr hohen Stufe gehalten werden. Wie wir früher gefunden haben ${ }^{2}$ ), lässt sich durch sorgfältigste Behandlung der durchschnittliche Fehler in der Bestimmung des zwischen Sehliessung und Öffnung verfliessenden Zeitraumes auf 0,00000129 Sek. herabdrücken. So gross war die Präzision bei den vorliegenden Untersuchungen nicht, da bei physiologischen Versuchen, wo durch die immer zu befürchtenden Veränderungen in der Erregbarkeit des Präparates grösste Eile geboten ist, die bei physikalischen Untersuchungen erlaubte bedächtige Arbeitsweise ausgeschlossen ist. Immerhin übersteigt der durchschnittliche Fehler der angegebenen Zeiten sicher nicht den Wert von 0,000004 Sek. Daraus ergibt es sich von selbst, dass die Dauer der kürzesten Stromstösse und -pausen nicht unter einen gewissen Wert herabsinken darf. Da wir nur sicheres Material mitteilen wollen, haben wir von allen Versuchen,

1) M. Gildemeister und O. Weiss, Pflüger's Arch. Bd. 94 S. 512.1903.

2) M. Gildemeister und O. Weiss; Annalen der Physik Bd. 17 S. 174. 1905. 
die unter 0,000012 Sek. herabgehen, diesen unsicheren Teil unberücksichtigt gelassen. Nebenbei wollen wir bemerken, dass es eine untere Grenze für die Dauer eines wirksamen Stromstosses nicht zu geben scheint, wenn die Intensität nur gross genug ist.

Bei so kurzen Zeiten könnte man Störungen durch Öffnungsfunken befürehten. Jedoch waren bei 4 Volt nie weniger als $5000.0 \mathrm{hm}$ im Kreise, wodurch derartige Störungen vollständig ausgeschlossen waren. Wie das in den Ann. d. Physik Bd. 17 S. 178 ahgedruckte Versuchsprotokoll zeigt, waren nennenswerte Deformationen der theoretischen Stromkurve nicht zu bemerken.

Wie es allgemein üblich ist, haben wir solche Reize als gleichwertig betrachtet, auf die der Muskel mit einer Minimalzuckung reagiert. Über diesen Begriff muss noch einiges gesagt werden.

Wenn man behauptet, dass ein gewisser Reiz $S$ eine Minimalzuckung hervorbringe, so will man damit sagen, dass ein um eine gewisse Grösse $\delta$ schwächerer nicht denselben Erfolg hat. Genau genommen kann man dann nur sagen, der Minimalreiz liege zwischen $S$ und $S-\delta$. Da über die Grösse von $\delta$ keine Vereinbarung besteht, ja sich gewöhnlich nicht einmal eine Angabe darüber findet - in vielen Fällen, wo es sich nicht um feinere quantitative Untersuchungen handelt, kommt es auch gar nicht so sehr darauf an --, ist die Bestimmung der Minimalzuckung mit einer gewissen Willkür behaftet. Deshalb haben wir hier einen vom allgemeinen Gebrauch etwas abweichenden $W{ }^{I}$ ) eingeschlagen: Zuerst wurde diejenige Reizstärke aufgesucht, durch die in zwei Versuchen zweimal eine möglichst kleine Zuckung zu erzielen war. Sodann wurde der Reiz so weit abgeschwächt, dass der Muskel zweimal hintereinander in Ruhe blieb. Beide Reizstärken finden sich in unseren Tabellen angegeben, ausserdem diejenigen (die natürlich $z$ wischen diesen beiden Werten liegen), die nur einmal von einer Zuckung begleitet waren, während diese das andere Mal ausblieb. Wenn $R$ Ruhe und $Z$ Zuckung bedeutet, ist es nun klar, was in den Tabellen die Überschriften $Z Z$, $Z R, R R$, besagen sollen.

Auf diese Weise haben wir also nicht bestimmte Werte für die Minimalreize gefunden, sondern obere und untere. Grenzen für dieselben. Da aber dadurch die subjektive Färbung der Resultate verschwindet, und da überdies die Grenzen gewöhnlich sehr nahe beieinander liegen, halten wir unser Verfahren für besser als das übliche.

1) In der Psychologie ist dies Verfahren ganz gebräuchlich. 


\section{Stromstösse.}

Hier stellten wir uns die Aufgabe, das Gesetz zu ermitteln, das zwischen der Intensität und der Dauer eines zur Erregung gerade ausreichenden Stromstosses besteht. Die Versuchsanordnung war dabei die folgende: Der von zwei Akkumulatorzellen gelieferte Strom wurde durch den Schliess- und den Öffnungskontakt des Pendels und durch zwei Rheostaten geleitet. Von den Endklemmen des einen Rhe0staten wurden Drähte zu den Elektroden abgezweigt. Durch Stöpselung der Rheostaten konnte die Spannungsdifferenz zwischen den Abzweigstellen dann in den Grenzen 0 und 4 Volt beliebig variiert werden.

Wir begnügten uns damit, die Spannungsdifferenz jedesmal zu bestimmen, ohne die Intensität des Nervenstromes festzustellen. Wenn der Nerv sich verhält wie ein selbstinduktions- und kapazitätsfreier Widerstand, so sind beide Grössen einander proportional. $\mathrm{Ob}$ diese Annahme bei kurzen Stromstössen zulässig ist, steht nicht fest. Indem wir diese Frage unentschieden liessen, beschränkten wir uns auf die Feststellung des Gesetzes zwischen Spannung und Dauer eines Minimalstromstosses.

Wenn die Rheostaten auf eine gewisse Spannung gestöpselt waren, suchten wir, wie schon eben gesagt, die minimale Dauer der wirksamen und die maximale des unwirksamen Stromstosses auf. Ausserdem wurde, wenigstens bei den ersten Versuchen, durch einfache Öffnung und Schliessung desselben Stromes ermittelt, welche Stufe des Zuckungsgesetzes vorlag. Später verzichteten wir auf diese Feststellung, weil das Präparat dadurch manchmal geschädigt wurde.

Die Versuche waren nicht alle von Erfolg begleitet. Hin und wieder sank die Erregbarkeit des Präparates sehr schnell, oder es trat Öffnungs- oder Schliessungstetanus ein. Wenn das der Fall war, wurde der Versuch von da an verworfen.

In folgender Tabelle 1 sind unsere Resultate mitgeteilt. In der ersten Spalte ist die Spannung $p$ in Volt angegeben, in der $\mathrm{z}$ w eite $\mathrm{n}$ die Dauer $t$ des eben wirksamen $(Z Z)$ und des eben unwirksamen $(R R)$ Stromstosses; $R Z$ bedeutet, wie schon gesagt, dass der Stromstoss einmal zu einer Zuckung führte, einmal nicht. Die Einheit der Zeit ist $\sigma \sigma$, d. h. ${ }^{1 / 1} 000000$ Sek. In der dritten Spalte findet sich das Produkt der Spalten 1 und 2, und zwar sowohl $p \times t_{R R}$ als auch $p \times t_{Z Z \text {. Wenn }} p$ proportional der Intensität $i$ des Nervenstromes ist, wie vorläufig angenommen werden soll, so sind die hier angegebenen Zahlen proportional der Elektrizitätsmenge, die den Nerven 
Über indirekte Muskelreizung durch Stromstösse und Strompausen. 333 durchströmt hat. Diese Spalte trägt deshalb auch die Überschrift Quantität, obgleich $p t$ von einer anderen physikalisehen Dimension ist als $i t$. Sollten spätere Untersuchungen zeigen, dass zwischen $p$ und $i$ andere Beziehungen bestehen, so sind entsprechende Korrektionen anzubringen.

Die letzte $S_{p}$ alte, überschrieben mit Energie $p^{2} t$, ist das Produkt der ersten und des rechten Teiles der dritten Spalte.

Tabelle 1 .

\begin{tabular}{|c|c|c|c|}
\hline $\begin{array}{c}\text { Volt } \\
p\end{array}$ & $\operatorname{Zeit}_{R R} t$ in $\sec ^{-6}$ & $\begin{array}{l}\text { Quantität } p t \\
R R \quad Z Z\end{array}$ & $\underset{Z Z}{\text { Energie }} p^{2} t$ \\
\hline
\end{tabular}

Versuch 2. Absteigender Strom. Elektrodendistanz $7 \mathrm{~mm}$. Nur Schliessungszuckung, keine Öffnungszuckung.

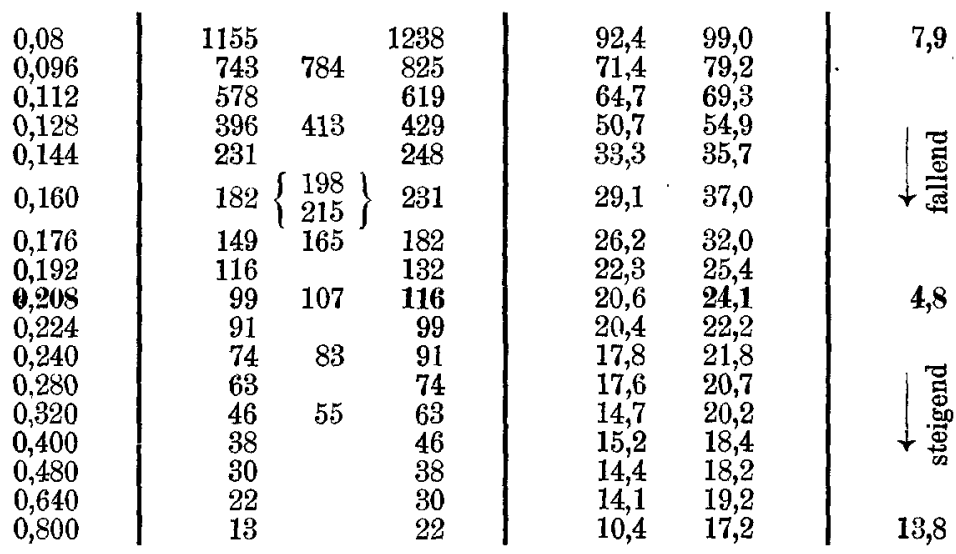

Versuch 5. Aufsteigender Strom. Elektrodendistanz $6 \mathrm{~mm}$. Schwellenreiz $\left.^{1}\right) 0,24$ Volt. Schliessungs- und Öffnungszuckung.

\begin{tabular}{|c|c|c|c|c|c|c|}
\hline $\begin{array}{l}0,28 \\
0,32\end{array}$ & $\begin{array}{l}736 \\
365\end{array}$ & & $\begin{array}{l}818 \\
406\end{array}$ & $\begin{array}{l}206,1 \\
116,8\end{array}$ & $\begin{array}{l}229,0 \\
129,9\end{array}$ & 64,1 \\
\hline 0,40 & 175 & $\left.\begin{array}{l}191 \\
208\end{array}\right\}$ & 224 & 70,0 & 99,6 & \\
\hline $\begin{array}{l}0,48 \\
0,56 \\
\mathbf{0 , 6 4} \\
0,72\end{array}$ & $\begin{array}{l}92 \\
68 \\
51 \\
35\end{array}$ & $\begin{array}{c}101 \\
76 \\
43\end{array}$ & $\begin{array}{r}109 \\
84 \\
59 \\
51\end{array}$ & $\begin{array}{l}44,2 \\
38,1 \\
32,6 \\
25,2\end{array}$ & $\begin{array}{l}52,3 \\
47,1 \\
37,8 \\
36,7\end{array}$ & 2 \\
\hline
\end{tabular}

Versuch 8. Aufsteigender Strom. Elektrodendistanz $4,5 \mathrm{~mm}$. Schwellenreiz ${ }^{1)} 0,104$ Volt. Nur Schliessungszuckung.

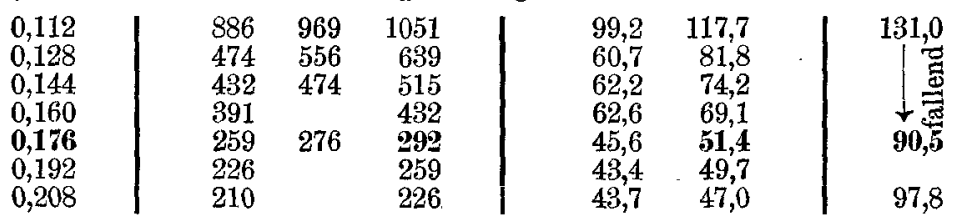

1) Für Schliessung eines Dauerstromes. 


\begin{tabular}{c|cc|c|c|c|}
\hline Volt & Zeit $t$ in $\sec ^{-6}$ & Quantitä $p t$ & Energie $p^{2} t$ \\
$p$ & $R R$ & $R Z$ & $Z Z$
\end{tabular}

Versuch 18. Aufsteigender Strom. Elektrodendistanz $10 \mathrm{~mm}$. Schwellenreiz $\left.{ }^{1}\right) \cdot 0,048$ Volt.

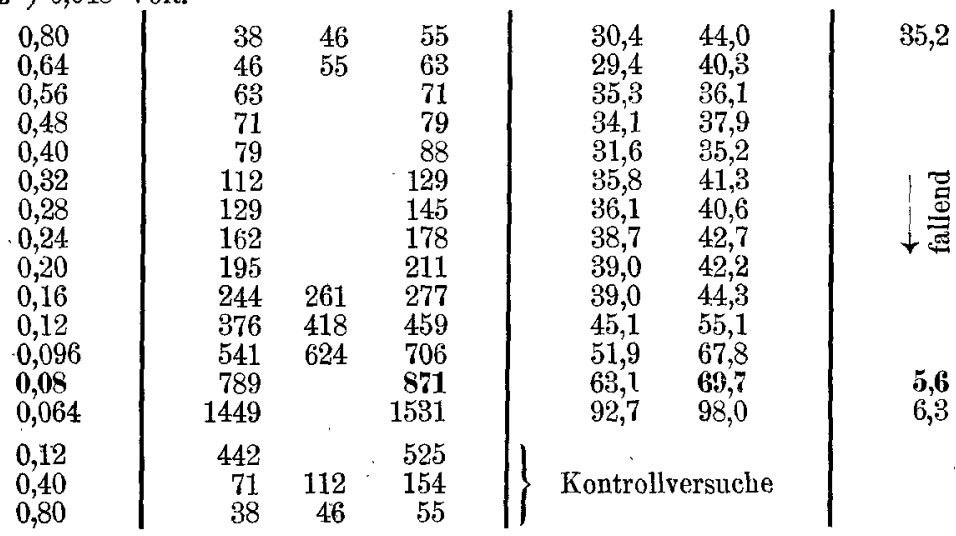

Versuch 21. Absteigender Strom. Elektrodendistanz $10 \mathrm{~mm}$. Schwellenreiz ${ }^{1)} 0,056$ Volt.

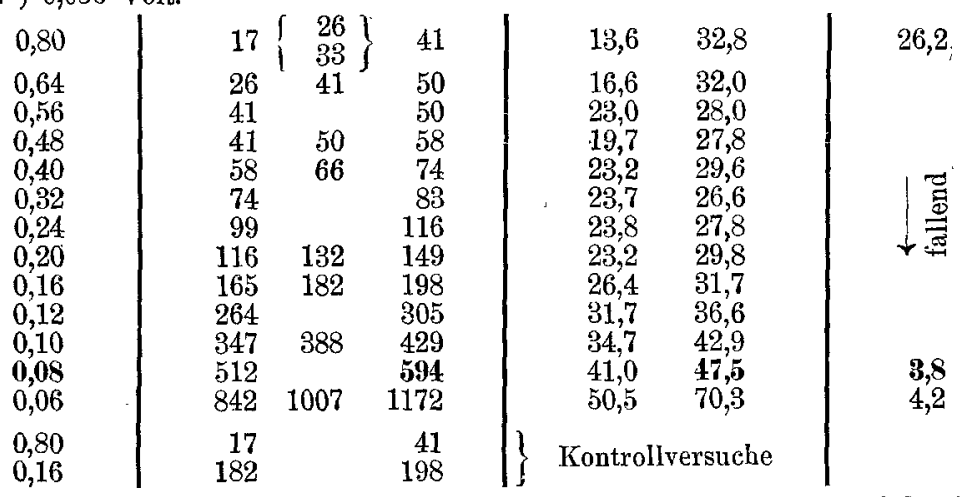

Versuch 22. Absteigender Strom. Elektrodendistanz $6 \mathrm{~mm}$. Schwellenreiz $\left.^{1}\right) 0,04$ Volt.

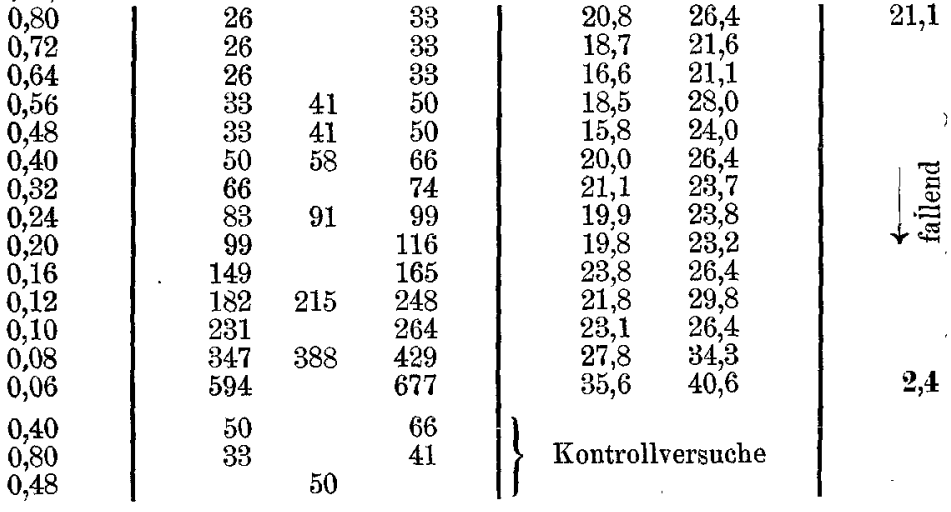

1) Für Schliessung eines Dauerstromes. 
Um das Zahlenmaterial übersichtlicher zu machen, trugen wir die Werte von $p$ und $t$ in ein Koordinatensystem ein. Da zeigte sich, dass die erhaltene Kurve fast genau mit einer gleichseitigen Hyperbel zusammenfällt, deren Asymptoten einerseits die $p$-Achse, andererseits eine zur $t$-Achse parallele Linie sind, etwa so, wie es die Fig. 1 zeigt. Wenn $b$ der Abstand der Parallelen von der $t$-Achse und $a$ eine zweite für jedes Präparat charakteristische Konstante bedeuten, so folgt daraus das gesuchte Gesetz

$$
(p-b) t=a \text {. }
$$

Durch eine kleine Umformung erhält man daraus das Gesetz in der auch von

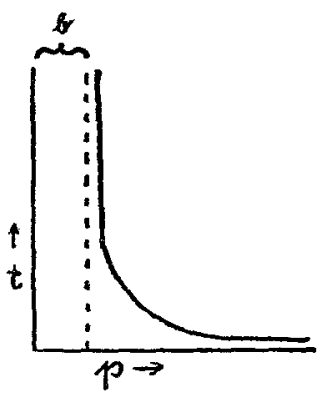

Fig. 1. G. Weiss ausgesprochenen Form $p t=a+b t$, oder, da $p t$ als Maass der Elektrizitätsmenge $Q$ betrachtet werden soll,

$$
Q=a+b t \text {. }
$$

Diese Formulierung ist insofern besser, als Abweichungen von diesem Gesetz, das durch eine gerade Linie dargestellt wird, ohne besondere Messung durch den blossen Angenschein festgestellt werden können. Deshalb haben wir hier den Wert oder vielmehr die beiden Grenzen für den Wert von $p t$ ausgereehnet und in der dritten Spalte angegeben.

Konstruiert man jetzt Kurven aus $p t$ und $t$, wie es in Fig. 2 (S. 336) für die Versuche 2 und 21 geschehen ist, so sieht man, dass das Gesetz von G. Weiss sich nicht in allen Fällen bewährt. Manchmal (Versuch 2) steigt die Quantität mit der Dauer des Stromstosses nicht geradlinig an, sondern in einer gegen die Zeitachse ein wenig konkaven Kurve, wie es ja auch schon von Lapicque gefunden ist. Versuch 21 dagegen zeigt keine wesentliche Krümmung. Die in der Tabelle 1 mitgeteilten Versuche verlaufen (ausser Versuch 2) alle wie Versuch 21 ; man findet bei 8 und 18 höchstens eine kleine Andeutung einer Krümmung. Bei anderen Versuchen, die wir aber wegen Erregbarkeitsänderungen nicht für zuverlässig halten, war die Krümmung deutlicher.

Von dem G. Weiss'schen Gesetze gibt es aber noch eine andere Abweichung, die unseres Wissens von anderer Seite noch nicht bemerkt ist und auch noch nicht bemerkt sein kann, weil noch niemand bisher mit so kurzen Stromstössen Versuche gemacht hat. 
Die $p t$-Kurve zeigt nämlich in ihrem Anfangsteil deutlich die Tendenz, der Zeitachse parallel zu werden. Das sieht man auf Fig. 2 an

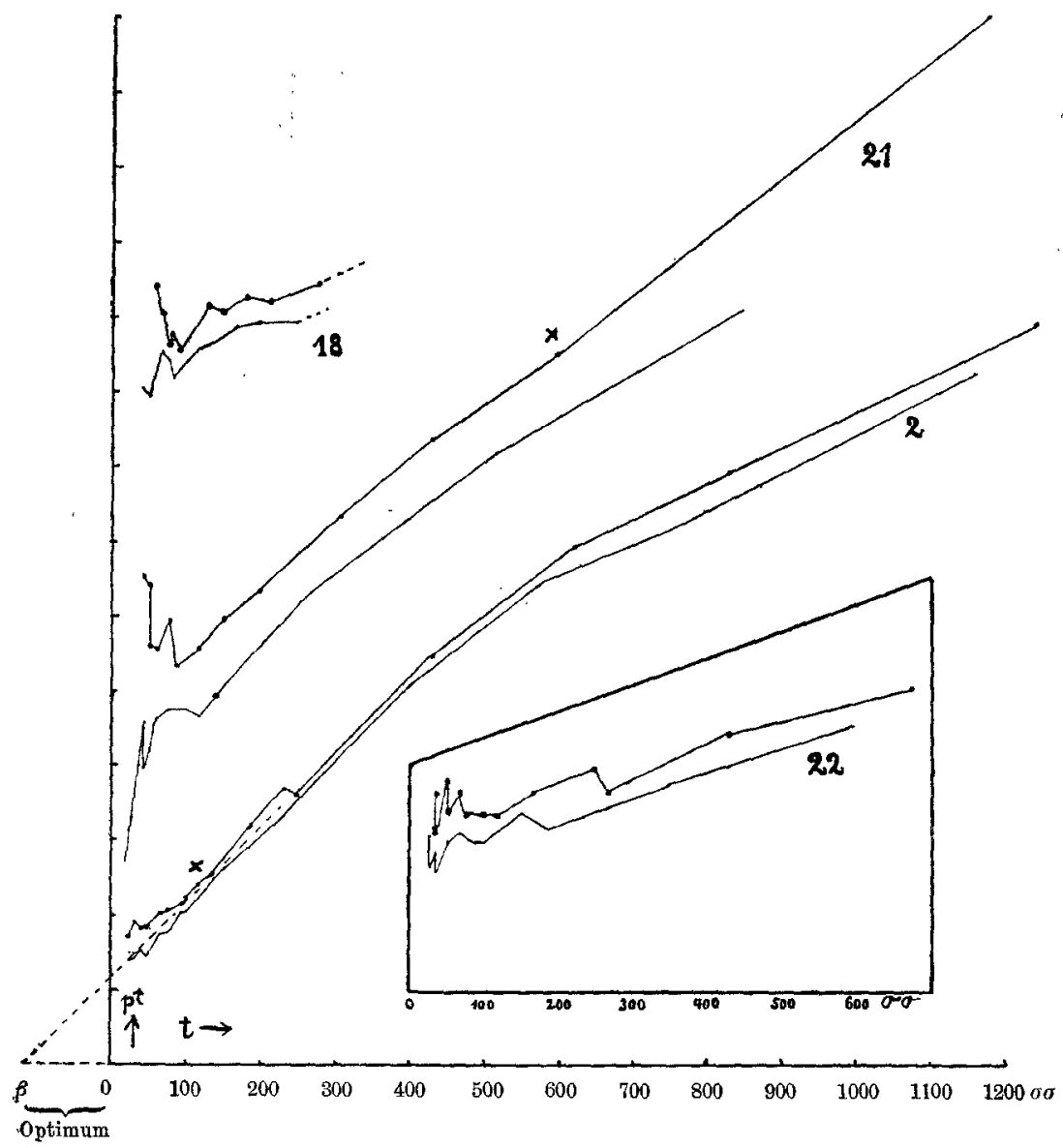

Fig. 2. Minimal-Stromstôsse. Die Ordinaten sind "Elektrizitätsmengen", die Abszissen "Zeiten" (Einheit $\sigma \sigma=1 / 1000000$ Sekunde). "Die Zahlen 2, 18, 21, 22 bezeichnen" die Versuchsnummer. Von jedem Kurvenpaar bedeutet der obere Linienzug den eben wirksamen, der untere den eben unwirksamen Stromstoss. $\times$ Stelle des Energieminimums.

Versuch 2 nur angedeutet (wobei der oberen Grenze natürlich das grössere Gewicht beizulegen ist); deutlicher ist es bei Versuch 22 und 18 (Fig. 2). Jedenfalls zeigt die $p t$-Kurve durchaus keine Tendenz, durch den Nullpunkt zu geben.

Dasselbe zeigt sich bei folgenden Versuchen, die in ihrem weiteren Verlaufe durch Störungen unbrauchbar wurden. 
Über indirekte Muskelreizung durch Stromstösse und Strompausen.

Tabelle 2.

\begin{tabular}{|c|c|c|c|c|c|}
\hline Volt & \multicolumn{3}{|c|}{ Zeit $t$ in $\sec ^{-6}$} & \multicolumn{2}{|c|}{ Quantität $p t$} \\
\hline$p$ & $R R$ & $R Z$ & $Z Z$ & $R R$ & $Z Z$ \\
\hline
\end{tabular}
zuckung

Versuch 9. Absteigender Strom. Schwellenreiz 0,092 Voit. Nur Schliessungs-

\begin{tabular}{|c|c|c|c|c|}
\hline $\begin{array}{l}0,32 \\
0,36 \\
0,40\end{array}$ & $\begin{array}{l}78 \\
61 \\
53\end{array}$ & & $\begin{array}{l}94 \\
78 \\
61\end{array}$ & $\begin{array}{l}25,0 \\
22,0 \\
21,2\end{array}$ \\
\hline 0,48 & 36 & $\left.\begin{array}{l}45 \\
53\end{array}\right\}$ & 61 & 17,3 \\
\hline $\begin{array}{l}0,56 \\
0,64\end{array}$ & $\begin{array}{l}28 \\
18\end{array}$ & $\begin{array}{l}36 \\
23\end{array}$ & $\begin{array}{l}45 \\
28\end{array}$ & $\begin{array}{l}15,7 \\
11,5\end{array}$ \\
\hline 0,72 & 23 & $\begin{array}{l}28 \\
38\end{array}$ & 38 & 16,6 \\
\hline $\begin{array}{l}0,80 \\
0,96\end{array}$ & $\begin{array}{l}18 \\
18\end{array}$ & $\begin{array}{l}23 \\
23\end{array}$ & $\begin{array}{l}28 \\
28\end{array}$ & $\begin{array}{l}14,4 \\
17,3\end{array}$ \\
\hline
\end{tabular}

Versuch 19. Absteigender Strom. Elektrodendistanz $12 \mathrm{~mm}$.

\begin{tabular}{|c|c|c|c|c|}
\hline $\begin{array}{l}0,80 \\
0,64 \\
0,56 \\
0,48\end{array}$ & $\begin{array}{l}38 \\
55 \\
71 \\
71\end{array}$ & 46 & $\begin{array}{l}55 \\
63 \\
79 \\
88\end{array}$ & $\begin{array}{l}30,4 \\
35.2 \\
39,8 \\
34,1\end{array}$ \\
\hline
\end{tabular}

Versuch 20. Absteigender Strom. Elektrodendistanz $16 \mathrm{~mm}$.

\begin{tabular}{l|rrr|rr}
0,80 & 50 & 58 & 66 & 40,0 & 52,8 \\
0,64 & 66 & & 74 & 42,2 & 47,4 \\
0,56 & 83 & & 91 & 46,5 & 51,0 \\
0,48 & 91 & & 99 & 43,7 & 47,5 \\
0,40 & 107 & 116 & 124 & 42,8 & 49,6 \\
0,32 & 140 & & 157 & 44,8 & 50,2
\end{tabular}

In Tabelle 3 sind die Zeiten, bis zu welchen die Elektrizitätsmenge einigermassen kunstant bleibt, zusammengestellt. Dabei sind auch die Tabelle 3.

\begin{tabular}{c|c}
\hline $\begin{array}{c}\text { Versuch } \\
\text { Nr. }\end{array}$ & $\begin{array}{c}t \\
\text { Einbeit } \text { sec }^{-6}\end{array}$ \\
\hline 2 & 74 \\
5 & 59 \\
18 & 277 \\
21 & 198 \\
22 & 264 \\
9 & 78 \\
19 & 88 \\
20 & 157
\end{tabular}
unteren Grenzen, die sich hier als sehr nützlich erweisen, gehörig berücksichtigt worden. - Man sieht, dass hier die Werte für $t$ ziemlich stark schwanken; aber bei physiologischen Versuchen sind feste Konstanten auch nicht zu erwarten.

Was die Energie anbetrifft, so hat diese ein deutliches Minimum (Tabelle 1, Spalte 4). Der zugehörige Stromstoss kann sehr verschiedene Länge haben $(60-870 \sigma \sigma)$. Die Energie der kürzesten Stromstösse, die wir angewendet baben, ist bis zu achtmal so gross als die optimale. Es gibt übrigens eine sehr einfache graphische Methode, um 
in den Kurven die Lage des Minimums zu bestimmen; verlängert man nämlich, wie es in Fig. 2 mit Versuch 2 geschehen ist, die $p^{t-}$ Kurve geradlinig nach links, bis sie die Abszissenachse links vom Nullpunkt 0 bei $\beta$ schneidet, so liegt das Minimum ebensoweit rechts von der Ordinatenachse wie $\beta$ links ${ }^{1}$ ).

Auf diese Weise kann man der Fig. 2 S. 336 entnehmen, dass bei Versuch 22 das Minimum höchstens erreicht, aber sicher nicht überschritten ist, was mit der letzten Spalte der Tabelle 4. Tabelle 1 übereinstimmt ${ }^{2}$ ).

\begin{tabular}{c|r}
\hline Volt & $\begin{array}{c}\text { Zeit } \\
\sigma \sigma\end{array}$ \\
\hline 0,048 & 3795 \\
0,064 & 3830 \\
0,080 & 2310 \\
0,096 & 2640 \\
0,120 & 2805 \\
0,160 & 1305 \\
0,200 & 810 \\
0,240 & 604 \\
0,320 & 183
\end{tabular}

Die mitgeteilten Versuche sind durchweg an solchen Präparaten angestellt, die schwachen Strömen gegenüber das normale Verhalten zeigten, d. h. nur auf Schliessung reagierten. In einem Falle untersuchten wir aber ein Präparat, das von schwachen Strömen nur bei Öffnung erregt wurde. Dieser Versuch, dessen Verlauf aus der Tabelle 4 zu entnehmen ist, scheint uns besonders bemerkenswert zu sein. Fr zeigt, dass ein Strom, dessen Öffnung erregen soll, yorher erst eine gewisse Zeit den Nerven durchflossen

1) Der Beweis ist leicht $2 u$ führen. Wenn $p=\frac{a}{t}+b$, so ist $p^{2} t=\frac{a^{2}}{t}+2 a b+b^{2} t$. Differenziert man diesen Ausdruck und setzt ihn $=0$, so hat man für das Minimum $t= \pm \frac{a}{b}$. Setzt man dagegen in der Gleichung $p t=a+b t, p t=0$, so bekommt man für den Schnittpunkt mit der $t$-Achse gleichfalls $t=-\frac{a}{b}$.

2) Der numerische Wert der Konstanten $a$ und $b$ der Gleichung $p t=a+b t$, ausgerechnet bei den Versuchen der Tabelle 1, ist recht verschieden. $a$ hat in den dortigen Einheiten etwa den Wert 15-30. Eigentlich bedeutet diese Grösse die Minimalquantität (für sehr kurze Stromstösse). Diese ist aber wegen des ejgentümlichen Anfangsverlaufs der Kurve in Wirklichkeit 10-20\% grösser.

$b$ soll den Grenzwert des $p$ für wachsendes $t$, d. h. für Dauerschliessung bedeuten. (Das ergibt sich aus der Gleichung $p=\alpha / t+b$, wenn man $t=\infty$ setzt.) Tatsächlich ist $a$ immer 10-20\% kleiner als der am Kopfe der Versuche angegebene Schwellenwert der Spannung. Da andererseits $b$ die Neigung der Turve $p t=a+b t$ bedeutet, und da diese, wie gesagt, für $t=\infty$ in Wirklichkeit grösser ist, als der Verlauf des untersuchten Teiles erwarten lässt, so-folgt daraus, das die pt-Kurve gegen die $t$-Achse konvex werden muss, wenn wir sie noch weiter nach rechts verfolgen. 
Über indirekte Muskelreizung durch Stromstösse und Strompausen. 339

haben muss. Diese Zeit ist desto länger, je schwächer der Strom ist, Bei 0,320 Volt wurde hier anch die Schliessung wirksam; die Folge davon ist, dass die Dauer des Stromstosses plötzlich sehr stark sinkt.

Die Stromstösse verhielten sich in jeder Beziehung wie andere elektrische Reize.' So werden sie bei Erwärmung des Nerven (im Ölhad) wirksamer, d. h. bei einer gewissen Spannung bedurfte es einer geringeren Dauer. Bei Abkühlung war es umgekehrt. Die Temperatur wurde dabei zwischen 11 und $31 \mathrm{Grad}$ variiert. Ferner wurde die minimale Dauer geringer, wenn die gereizte Stelle in Katelektrotonus versetzt wurde, während der anelektrotonische $\mathrm{Zu}$ stand entgegengesetzt wirkte.

In anderen Versuchen steigerten wir die Stromdauer bei konstanter Spannung über die minimale hinaus. Auch dann zeigten sich keine Unterschiede gegenüber anderen Reizen: die Zuckungsdauer wuchs erst schneller, dann langsamer zu einem Maximum. Die Maximalzuckung trat ein, wenn das $(1,3-) 2(-3)$ fache der Minimaldauer erreicht war. Hätten wir noch länger dauernde Ströme benutzt, so hätten wir das Gebiet der Fick'schen Lücke ${ }^{1}$ ) erreichen müssen; zu so langen Schliessungen war aber unser Apparat damals nicht eingerichtet.

Nebenbei mag erwähnt werden, dass die Figur auf Seite 46 der Fick'schen Arbeit, die auch die Beziehung zwischen Intensität und Stromdauer darstellt, mit unserer Figur 1 auf Seite 335 grosse Ähnlichkeit hat. Daraus könnte man schliessen, dass dieser Forscher schon das G. Weiss'sche Gesetz gekannt hat. Klar formuliert hat er es nicht, wie überhaupt der damaligen Zeit genauere quantitative Untersuchungen fern lagen.

\section{Strompansen.}

In diesem Teil unserer Untersuchung sollte festgestellt werden, ob auch zwischen der Dauer einer eben zur Erregung führenden Lücke in einem konstanten Strom und der Intensität dieses Stromes eine einfache Beziehung bestände. Wir erwarteten ein ähnliches Gesetz zu finden, wie es sich bei Stromstössen gezeigt hatte, da der zu untersuchende Vorgang ja auch so aufgefasst werden kann, als ob

1) Adolf Fick, Untersuchungen über elektrische Nervenreizung. Braunschweig 1864. 
durch dieselbe Nervenstrecke ein kurzer Strom entgegengesetzter Richtung, aber gleicher Intensität geschickt wird, der sich mit dem Dauerstrom zu Null ergänzt. Jedoch waren auch beträchtliche $A b$ weichungen nicht ausgeschlossen; denn die Kathode dieses fingierten Stromstosses fällt ja mit der Anode des polarisierenden Stromes zusammen.

Die Versuche verliefen in folgender Weise: Zuerst wurde mit der Hand ein Schlüssel in Tätigkeit gesetzt, so dass der Strom in den Nerven hereinbrach. Nach genau einer Sekunde fiel das Pendel und schloss eine gutleitende Nebenschliessung zum Nerven, die nach sehr kurzer Zeit durch den Öffnungskontakt wjeder unterbrochen wurde.

Alle unsere Angaben beziehen sich auf den Intervall von 1" zwischen Strombeginn und Pause. Verkürzung dieses Zeitraumes scheint die Pause wirksamer zu machen und umgekehrt. Jedoch fehlen uns ausgedehntere Versuche über diesen Punkt.

Unsere Ergebnisse finden sich in der Tabelle 5. Der Einfachheit halber ist hier die Vorstellung eines Stromstosses festgehalten worden; in diesem Sinne sind die Überschriften Quantität und Energie zu verstehen. Wie bei den Stromstössen ist auch hier angenommen worden, dass die Intensität der Spannungsdifferenz proportional sei. Über diesen Punkt haben wir uns auf S. 332 ausgesprochen.

Tabelle 5 .

\begin{tabular}{c|ccc|cc|c|c}
\hline Volt & \multicolumn{2}{|c|}{ Zeit $t$ in $\sec ^{-6}$} & Quantität $p t$ & Energie $p^{2} t$ & Zuk- \\
$p$ & $R R$ & $R Z$ & $Z Z$ & $R R$ & $Z Z$ & $Z Z$ & $Z$ kungen $)$ \\
\hline
\end{tabular}

Versuch 10. Aufsteigender Strom.

\begin{tabular}{|c|c|c|c|c|c|c|}
\hline $\begin{array}{l}0,08 \\
0,12 \\
0,16 \\
0,24 \\
0,32 \\
0,40 \\
0,56 \\
0,80\end{array}$ & $\begin{array}{r}1155 \\
660 \\
289 \\
198 \\
182 \\
149 \\
116 \\
(41)\end{array}$ & $\begin{array}{l}198 \\
165\end{array}$ & $\begin{array}{r}1238 \\
784 \\
330 \\
215 \\
215 \\
182 \\
132 \\
(50)\end{array}$ & $\begin{array}{c}92,4 \\
79,2 \\
46,2 \\
47,5 \\
58,2 \\
59,6 \\
65,1 \\
(32,8)\end{array}$ & $\begin{array}{c}99,0 \\
94,1 \\
52,8 \\
51,0 \\
68,9 \\
72,8 \\
73,9 \\
(40,0)\end{array}$ & 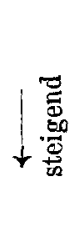 \\
\hline
\end{tabular}

Versuch 11. Aufsteigender Strom.

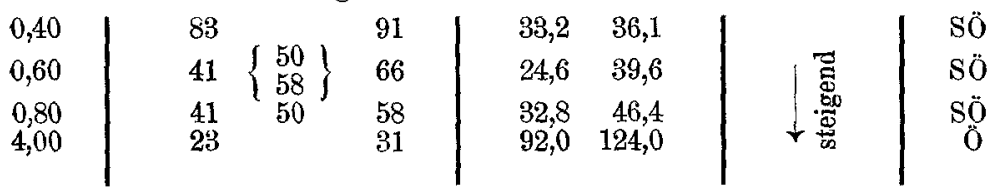

1) S. Schliessungszuckung, $\ddot{0}$. Öffnungszuckung. 
Über indirekte Muskelreizung durch Stromstösse und Strompausen. 341

\begin{tabular}{|c|c|c|c|c|c|c|}
\hline Volt & \multicolumn{2}{|c|}{ Zeit $t$ in $\sec ^{-6}$} & \multicolumn{2}{|c|}{ Quantität $p t$} & \multirow{2}{*}{$\begin{array}{c}\text { Energie } p^{2} t \\
Z Z\end{array}$} & \multirow{2}{*}{$\begin{array}{c}\text { Zuk- } \\
\text { kungen }\end{array}$} \\
\hline$p$ & $R R$ & $R Z$ & $R R$ & $Z Z /$ & & \\
\hline
\end{tabular}

Versuch 12. Aufsteigender Strom.

\begin{tabular}{|c|c|c|c|c|c|c|}
\hline 0,40 & 59 & & 76 & 23,6 & 30,4 & \\
\hline 0,32 & 125 & & 142 & 40,0 & 45,4 & \\
\hline 0,24 & 258 & & 291 & 61,9 & 69,8 & \\
\hline 0,16 & 802 & 2617 & 884 & 128,3 & 141,4 & . \\
\hline 0,12 & 2452 & 2782 & 3112 & 174,2 & 373,4 & \\
\hline
\end{tabular}

Versuch 13. Absteigender Strom.

\begin{tabular}{|c|c|c|c|c|c|c|}
\hline 0,08 & 5074 & & 5404 & 405,9 & 432,2 & 34,6 \\
\hline 0,12 & 1774 & 1939 & 2104 & 212,8 & 252,5 & 30,3 \\
\hline 0,16 & 866 & & 949 & 138,6 & 151,8 & $\mathbf{2 4}, 3$ \\
\hline 0,24 & 413 & & 454 & 99,1 & 109,0 & 26,2 \\
\hline 0,32 & 248 & & 289 & 79.4 & 92,5 & 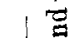 \\
\hline 0,40 & 190 & & 206 & 76,0 & 82,4 & 5 \\
\hline 0,56 & 107 & & 124 & 59,9 & 69,4 & $\downarrow$ \\
\hline 0,80 & & & 83 & 59,2 & 66,4 & 点 \\
\hline 4,00 & 83 & $\begin{array}{r}99 \\
107\end{array}$ & 124 & 332,0 & 496,0 & 198,4 \\
\hline
\end{tabular}

Versuch 14. Absteigender Strom,

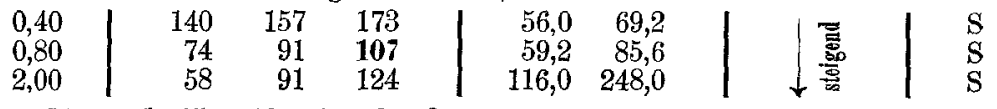

Versuch 15. Absteigender Strom.

\begin{tabular}{|c|c|c|c|c|c|c|c|}
\hline 0,16 & 1939 & & 2104 & 310,2 & 336,6 & 53,9 & $\mathrm{~S}$ \\
\hline 0,20 & 949 & & 1114 & 189,8 & 222,8 & 44,6 & s \\
\hline 0,24 & 701 & & 784 & 168,2 & 188,2 & 45,2 & $\mathrm{~S}$ \\
\hline 0,32 & 413 & & 454 & 132,2 & 145,3 & 46,5 & S \\
\hline 0,40 & 190 & 248 & $2 \varangle 9$ & 76,0 & 115,6 & 46,2 & $\tilde{S}$ \\
\hline 0,56 & 157 & 173 & 190 & 87,9 & 106,4 & O & $\mathrm{S}$ \\
\hline 0,80 & 107 & & 124 & 85,6 & 94,2 & 承 & $\mathrm{S}$ \\
\hline 0,32 & {$[413$} & 454 & 495 & $\leftarrow$ Kontr & ollversuch & & $\approx$ \\
\hline 2,00 & 74 & 83 & $\$ 1$ & 148,0 & 182,0 & $\downarrow \underset{\omega}{\Phi}$ & sö \\
\hline 4,00 & 91 & 107 & 124 & 364,0 & 496,0 & 1984,0 & $\left.\mathrm{Sö}^{1}\right)$ \\
\hline
\end{tabular}

Versuch 16. Aufsteigender Strom.

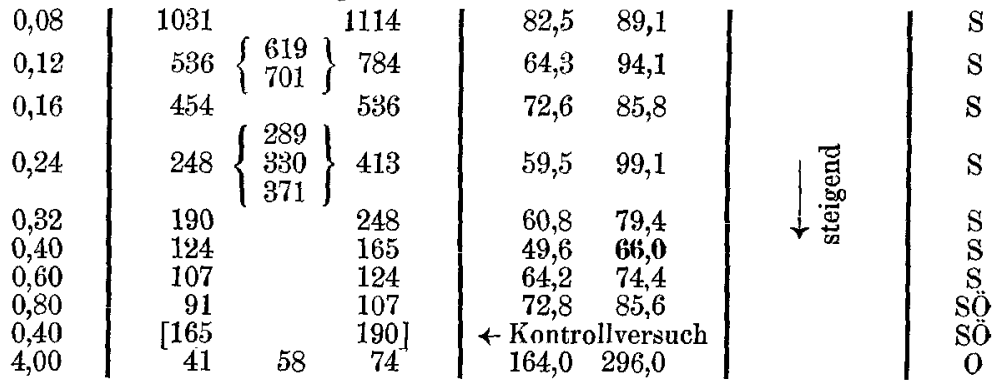

Versuch 17. Absteigender Strom.

$\begin{array}{llllllllll}2,00 & \text { I } & 41 & 50 & 58 & \text { I } & 82,0 & 116,0 & \text { | }\end{array}$

1) Sehr kleine Öffnungszuckung. 
Diese Zablen sind nicht ganz leicht zu übersehen. Es machen sich offenbar zwei Einflüsse geltend, die bei den Stromstössen nicht zu bemerken waren: erstens die Stromrichtung, zweitens die elektrotonisierenden Wirkungen des Dauerstromes.

Während ein Stromstoss, d. h. eine Schliessung und eine gleich darauf folgende Öffnung, einen unveränderten Nerven trifft, ist der Vorgang hier ganz anders. Hier wird der Nerv erst der Wirkung eines eine Sekunde lang fliessenden konstanten Stromes unterworfen, wodurch bekanntlich seine Erregbarkeit sich ändert. Dann wird der polarisierende Strom unterbrochen und kurz darauf wieder geschlossen. Es kommt also darauf an, welche Frregbarkeit er bei der zweiten Schliessung an der Kathode antrifft, ob die Leitungsfähigkeit nicht vorher gelitten hat usw. Man wird also die beiden Stromrichtungen und bei diesen wieder die drei Stufen des P fl üg e r schen Zuckungsgesetzes gesondert ins Auge fassen müssen.

a) Aufsteigender Strom (Versuche 10,11,12, 16). Mit steigender Spannung nimmt die minimale Dauer des erregenden Stromstosses ab. Das gilt für alle drei Stufen des Zuckungsgesetzes. Ein Vergleich mit Tabelle 1 S. 333 lehrt, dass bei gleicher Spannung eine erregende Pause durchschnittlich drei- bis viermal so lange dauern muss als ein Stromstoss.

Die Quantität nimmt nicht wie bei den Stromstössen mit wachsender Spannung $a b$, sondern sie hat bei mittlerer Spannung 0,24-0,40 Volt) ein Minimum. Versuch 11 und 12 zeigen, wie sie von 0,40 Volt zu höheren und niederen Spannungen hin zunimmt. (Den Schluss des Versuches 10 möchten wir ausser Betrachtung lassen, weil das Präparat anscheinend geschädigt war. Das zeigte sich daran, dass es kurz darauf in Öffnungstetanus verfiel). Welche Stufe des Zuckungsgesetzes erreicht ist (Versuch 16, alle drei Stufen), scheint dabei gleichgültig zu sein.

Die Energie scheint mit steigender Spannung zuzunehmen. Freilich macht davon Versuch 12 eine Ausnahme. Unser Material reicht nicht aus, um diesen Punkt sicherzustellen.

$\mathrm{Ob}$ die Pause immer weiter sich verkürzt, wenn die Spannung über 4 Volt hinaus gesteigert wird, darüber fehlen uns Versuche.

b) Absteigender Strom (Versuche 13, 14, 15, 17). Auch hier nimmt die Pausenlänge mit wachsender Spannung ab, aber nur bis zu einer gewissen Grenze. Bei sehr starken Strömen nimmt 
sie wieder zu; sie hat also ein Minimum. Wahrscheinlich hängt das mit dem Fortfall oder dem Kleinerwerden der Öffnungszuckung zusammen (Versuch 15).

Die Quantität hat anch ein Minimum, das nicht mit dem Zeitminimum zusammenzufallen braucht (Versuch 15).

Ebenso durchläuft die Energie ein Minimum, das bei ziemlich niedrigen Spannungen liegt (Versuche 13 und 15, 0,16 und 0,20 Volt).

Die Analyse der Tabelle 5 lehrt, dass man auch bei graphischer Veranschaulichung der Versuche zwischen den verschiedenen Stufen des Zuckungsgesetzes wird unterscheiden müssen. Die einfachsten Verbältnisse sind bei der ersten Stufe zu erwarten. Deshalb sind die Versuche 12, 13 und 15 in Fig. 3 dargestellt mit Weglassung der Werte für 2 und 4 . Volt, und zwar wieder wie in Fig. 1, die Quantität als Funktion der Zeit. Die unteren Gręzwerte sind fortgelassen. (In Versuch 16 ist die Erregbarkelt offenbar nicht konstant geblieben, wie der regellose Gang der dritten Spalte zeigt.)

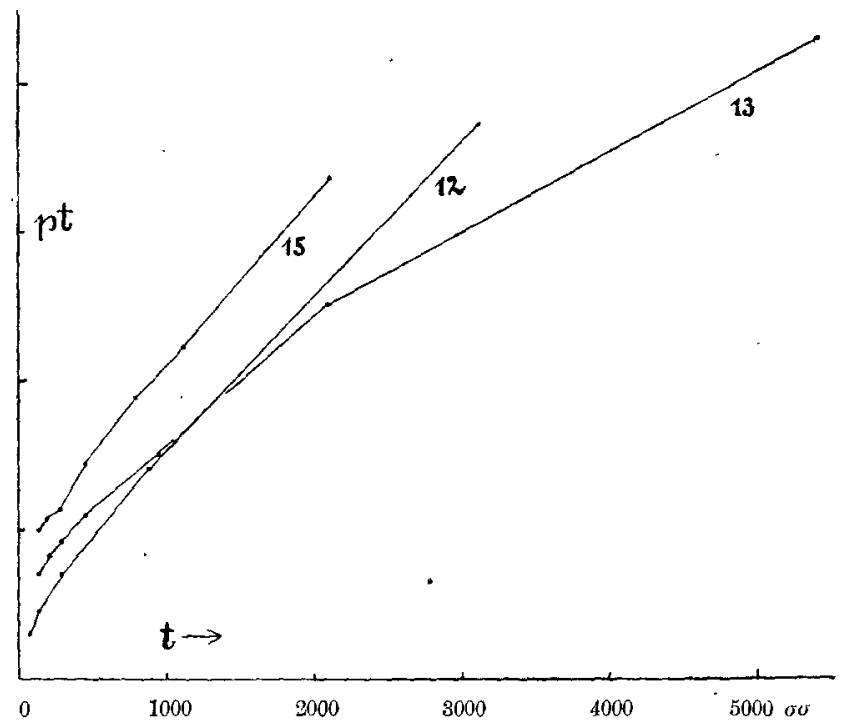

Fig. 3. Minimal-Strompausen. Die Ordinaten bedeuten "Elektrizitätsmengen", die Abszissen "Zeiten" (Einheit $\sigma \sigma=1 / 100000$ Sekunde). Die Zahlen 12, 13 und 15 sind Versuchsnummern (s. Tabelle 5).

Man sieht denn auch, dass die Quantitätskurve eine einfache Form hat, deren Ähnlichkeit mit den Stromstosskurven in die Augen springt. Auf eine mathematische Formulierung, die auch der leichten Krümmung Reehnung trägt, verzichten wir; nach 
unserer Meinung genügt die Feststellung, dass die Kurve eine geringe, aber gesetzmässige Abweichung von der Geraden aufweist.

An den mitgeteilten Tatsachen lässt sich einigermaassen ein Bild von der Erregbarkeitsänderung an den Elektroden gewinnen. Wollten wir uns aber darüber aussprechen, so würden wir damit den Boden der Tatsachen verlassen und unserem Vorbaben, hier von allen theoretischen Erörterungen abzusehen, untreu werden. Deshalb verschieben wir das auf eine spätere Gelegenheit.

Zum Schlusse wollen wir noch erwähnen, dass wir wie die Stromstösse, so auch die Pausen über die Minimaldauer verlängert haben. Dann wuchs auch hier die Grösse der Zuckung, um bei schwachen Strömen in der $1,5-$ bis 3 fachen Zeit das Maximum zu erreichen. Bei starken Strömen jedoch (1 Volt und mehr) war das Maximum manchmal selbst bei zehnfacher Dauer nicht zu erzielen.

\section{Zusammenfassung.}

Mit Hilfe eines Helmholtz'schen Pendels wurden Nervmuskelpräparate vom Frosch durch kurze Stromstösse und Strompausen gereizt, deren Zeitdauer bis auf $13 / 1000000$ Sekunden herabging. Die Spannung des Reizstromes wurde dabei zwischen 0,08 und 4 Volt variiert.

Um jede Willkür in der Bestimmung des Minimalreizes auszuschliessen, wurde dieser zwischen zwei Grenzen eingeschlossen. Es wurden also bestimmt: erstens ein eben wirksamer Reiz, zweitens ein möglichst in der Nachbarschaft des ersten liegender, unwirksamer. Der wahre Minimalreiz liegt dann zwischen beiden.

Bei Stromstössen bewährte sich innerbalb gewisser Grenzen das (uns zur Zeit dieser Untersuchung unbekannte) G. Weiss'sche Gesetz, dass die Elektrizitätsmenge aus zwei Teilen besteht, einem konstanten und einem proportional der Stossdauer zunehmenden. Abweichungen von diesem Gesetz zeigten sich darin, dass erstens die Quantität manchmal mit der Zeit nicht geradlinig, sondern ein wenig konkav gegen die Abszissenachse ansteigt. Zweitens ist sie für sehr kurze Stromstösse (von der Grössenordnung 100-250 $\mathrm{sec}^{-6}$ ) anscheinend konstant. Die Energie durchläuft ein sehr a usgeprägtes Minimum.

Bei Strompausen gilt für nicht zu starke Ströme der von uns schon früher ausgesprochene Satz: „In einem stärkeren Strom 
Über indirekte Muskelreizung durch Stromstösse und Strompausen. 345 bedarf es zu demselben Erregungseffekte einer kürzeren Pause als in einem schwächeren." 1 ) Diese Regel hat nur bei hohen Spannungen (über 2 Volt) und absteigendem Strom eine Ausnahme; in diesem Falle nimmt die Dauer wieder etwas zu, so dass ein Minimum existiert.

Betrachtet man die Pausen als (negative) Stromstösse, die sich einem Dauerstrom superponieren, so kann man auch hier von Quantität und Energie sprechen. Die Quantität hat bei beiden Stromrichtungen ein Minimum, das ungefähr der Intensität zukommt, bei der die mittlere Stufe des Zuckungsgesetzes beginnt. - Die Energie durchläuft anscheinend ebenfalls bei beiden Stromrichtungen ein Minimum.

Eine Pause muss, um zu wirken, etwa drei- bis viermal so lang sein als ein Stromstoss gleicher Intensität.

Die Kurve, welche die Quantität einer Minimalpause als Funktion ihrer Dauer darstellt, hat bei schwachen Strömen, unabhängig von der Richtung, Ähnlichkeit mit der analogen bei Stromstössen. Auch sie steigt mit wachsender Dauer und lässt (im Gegensatz zu den Stromstössen nicht nur manchmal, sondern immer) eine schwache konkave Krümmung gegen die Abszissenachse erkennen.

Theoretische und historisehe Bemerkungen über die Befunde sollen an anderer Stelle gemacht werden.

1) M. Gildemeister, l. c. 